Chrischta Ganz Solothurn die 6. Fortbildungstagung für Traditionelle Chinesische Medizin (TCM) statt. Der grösste TCM-Kongress der Schweiz, unterdessen zum zweitgrössten europäischen TCMKongress gewachsen, wurde von der Europäischen Akademie für TCM (EATCM) mitgetragen und stand unter dem Tagungsmotto «Die Lebensphasen - Die ersten 40 Jahre». Schwerpunkt des Kongresses war ein Thema, das wortwörtlich mitten aus dem Leben kommt: Sterilität und Infertilität. Unerfüllter Kinderwunsch ist eine Thematik, die viele aus der täglichen Praxis kennen. Die Teilnehmenden erwartete ein spannendes Programm aus wissenschaftlichen und praxisorientierten Präsentationen und Workshops mit nationalen und internationalen Experten: Paul Ulrich Unschuld (Berlin), Frank Bahr (München), Frank He (Santa Cruz), Christine Bodenschatz-Li (Hamburg), Yaron Seidmann (Greenwich) und Martine Depondt-Ghadet (Paris), um nur einige zu nennen.

Das Präsidium des Kongresses haben seit diesem Jahr Dr. Anita Meyer (FMH Allgemeine Innere Medizin)

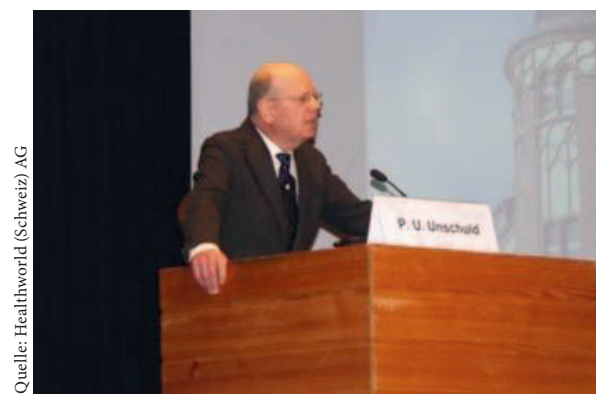

Abb. 1. Einen Einblick in die Vergangenheit gab Prof. Dr. Paul Unschuld in seinem Referat.

\title{
Bericht zum 6. ASA TCM-Kongress in Solothurn
}

und Simon Becker (dipl. Akupunkteur und Herbalist SBO-TCM) von Dr. Jörg Fritschi (FMH Allgemeine Innere Medizin, FACP) übernommen. Jörg Fritschi darf gerne als «Vater des ASA TCM-Kongresses» bezeichnet werden und ihm sei an dieser Stelle für sein Engagement herzlich gedankt.

Den Auftakt machte Prof. Dr. Paul Ulrich Unschuld (Abb. 1) mit seinem Hauptreferat zum Thema «Einblick in die Vergangenheit - was kann sie uns bieten?». Prof. Unschuld, Experte für die Medizingeschichte, legt den Fokus seines wissenschaftlichen Wirkens auf den Vergleich von Geschichte, Theorie und Ethik der Medizin in Europa und in China sowie die Integration nichtwestlicher Heilsysteme in unser Gesundheitssystem. Einigen der Leser und Leserinnen dürfte Unschuld durch einen Artikel im TagesAnzeiger ( $w w w$.tagesanzeiger.ch) im letzten Mai zum Thema «Die Traditionelle Chinesische Medizin ist in Wirklichkeit ein Kunstprodukt» bekannt sein. Aufgrund des grossen Echos darauf (um es nicht Aufschrei zu nennen) ist es besonders spannend und von der Kongressleitung ebenso mutig wie gut gewählt, Prof. Unschuld als Referenten an die Tagung einzuladen.

Unschuld stellte die Frage in den Raum, inwieweit sich Heilkunst und Medizin voneinander unterscheiden - eine aufschlussreiche Frage, da ja in der deutschen Sprache beide Wörter bekannt sind und dementsprechend auch in ihrem jeweiligen Kontext verwendet werden. Unschuld bezeichnete Heilkunst als nichtmedizinische Kunst, die vor und zeitgleich mit me- dizinischer Heilkunst existiert. Die Tatsache, dass am diesjährigen Kongress auch Christine Bodenschatz-Li das Thema aufnahm, zeigte deutlich die Notwendigkeit, sich als Akteur im Gesundheitssystem die Frage zu stellen, «wie man das Heilen lernen kann».

Beginnend mit dem 2. Jahrhundert v. Chr. entsteht ein neues Verständnis von Krankheit und Gesundheit auf der Grundlage säkularer Naturwissenschaft. Der Organismus wird als Ganzes verstanden und interagiert in komplexer Weise mit einzelnen Funktionszentren, die untereinander über den Austausch von Blut und Qi über das System von Meridianen verbunden sind. Laut Unschuld sind die YinYang- und die Fünf-Phasen-Lehre als Kunstprodukt bewusst gebildet worden. Dem Entstehungsprozess liegt die Erfahrung «Die Existenz ist ein ewig währender Kampf» (siehe hierzu Suwen 69: «Wenn die Qi einander überwinden, das ist Harmonie. Wenn sie sich nicht gegenseitig überwinden, das ist Krankheit.») zugrunde, was nach jahrhundertelangen kriegerischen Auseinandersetzungen in China auch verständlich erscheint. Das Erklärungsmodell kann also durchaus auch als Anleitung verstanden werden, wie ein Überleben in einer Welt, die von ewig währendem Kampf und von Vernichtung geprägt ist, überhaupt möglich ist. Man macht sich damit das Wissen über den Kampf zunutze und übersetzt es in die Lebensgestaltung. Die Botschaft des neuen Verständnisses von Gesundheit und Krankheit formuliert Unschuld in drei Grundaussagen:

- Es gibt Naturgesetze.

\section{KARGER}

Fax +497614520714 Information@Karger.com www.karger.com
(๑) 2013 S. Karger GmbH, Freiburg
Chrischta Ganz

Praxis für Naturheilkunde

Steinentischstrasse 1, 8002 Zürich, Schweiz

naturheilpraxis@chrischtaganz.ch 
- Ihre Befolgung garantiert ein langes Leben.

- Niemand muss sich mehr vor Dämonen, Ahnen und Göttern fürchten.

So entsteht die Lebenshaltung einer existenziellen Selbstbestimmung, wunderbar ausformuliert in «Mein Schicksal liegt in meinen Händen, nicht im Himmel» (Tao Hongjing, ca. 500 v. Chr.). Damit ist es der Mensch, der die Geister kontrolliert und nicht umgekehrt! Im konkreten Gesundheitsverständnis heisst das dann: «Blut und Qi sind die Geister im Menschen, sie muss man sorgfältig nähren» (Suwen 26). Der Körper ist ein politischer Organismus, der sich aus verschiedenen Regionen zusammensetzt, die jeweils über ein Verwaltungszentrum, einen Gouverneur und einen Untergebenen verfügt. (Inwiefern die heute wichtigen Aussagen aus der TCM strategisch ausgewählt wurden, bleibe dahingestellt. Man ist nach solchen Überlegungen durchaus geneigt, bei Suwen die Botschaft «Benehmt euch nach den Gesetzen, macht keine Opposition, sonst werdet ihr krank» herauszuhören.) Was in der Gesellschaft ein komplexes System von Menschen, Transportwegen und Gütertransfer ist, entspricht im menschlichen Organismus dem komplexen System des Austauschs von Blut, Qi und Leitbahnen. Therapie bedeutet in diesem Sinne ein Ordnen der Strukturen. Während Konfuzianer und Legalisten das Versprechen vertreten, dass Krankheit vermeidbar ist und nur aufgrund von menschlichem Fehlverhalten entsteht, halten Daoisten aus ihrer Beobachtung der Natur die Tatsache fest, dass der Körper an sich dem Verfall unterliegt und somit eine unvermeidbare Ursache von Krankheit ist.

Unschuld analysierte eine immense Anzahl an handschriftlichen chinesischen Büchern zur Heilkunde (im Frühling 2012 ist Unschulds Katalog von fast 900 handschriftlichen Büchern chinesischer Ärzte der letzten
400 Jahre erschienen), in denen er keine Spur von Yin-Yang- und Fünf-Phasen-Verständnis fand. Was man heute in Europa unter TCM versteht, scheint von einer Elite in China geprägt und keineswegs für die breite chinesische Bevölkerung repräsentativ zu sein. Über tausend Jahre scheinen zwei Traditionen nebeneinander bestanden haben, zum einen die Suwen-Medizin mit ihrer Gefässtheorie, der Akupunktur und der Diätetik und zum anderen die Bencao-MateriaMedica-Arzneikunde mit den pharmazeutischen Drogen.

Wichtig ist zu bedenken, dass wir Europäer das chinesische System nach unserem eigenen Weltverständnis interpretieren. Die ersten TCMBücher wurden somit auch von westlichen Besuchern geschrieben, aufbauend auf westlichem Gedankengut. So klingt zwar einiges fremdartig für uns, aber gleichzeitig auch vieles vertraut. Die menschliche Suche nach Erklärungsmodellen zu Leben, Tod, Wandel, Gesundheit und Krankheit ist auf der ganzen Welt sichtbar und insbesondere in jedem systemischen Denken zu finden. Dass sich das systemische Denken in der Prävention und Behandlung von Krankheiten bewährt, steht ausser Frage.

Den ersten Kongresstag bereicherte zudem Dr. Martine DepondtGadet, Ärztin, diplomierte Akupunkteurin, Homöopathin und Expertin für Qi Gong, mit ihrer Qi-GongEinführung im Mittagsintermezzo und ihrem Referat «Stérilité et Infertilité». Weiterhin wurden den Teilnehmenden Seminare und Tageskurse über Aurikulotherapie, TCM in der Sportmedizin, Qi Gong bei Unfruchtbarkeit, die Behandlung von Endometriose, aber auch aktuelle Studienergebnisse zur Akupunktur bei unerfülltem Kinderwunsch geboten.

Dr. med. Ansgar Römer eröffnete den zweiten Kongresstag mit seinem Referat zu seinen Erfahrungen mit der Akupunktur in der Geburtshilfe. Im
Anschluss daran folgten spannende Seminare zum Thema «Ernährung für Frauen unter 40 Jahren», zu «Mutter und Kind», Pediatric Tuina, Stressprävention durch Tai Ji und vieles mehr.

Christine Gabriel (Aachen) stellte in ihrer Präsentation «den Durchgang durch die fünf Wandlungsphasen von der Empfängnis bis zur Geburt» vor. Der erste und der zweite Monat unterliegen der Wandlungsphase $\mathrm{Holz}$ (Le/Gb); die Implantation und die Bildung der drei Keimblätter stehen an. Shi Pei zeigt den Beginn des Embryos. Es ist der Anfang des Wassers, Yin und Yang vereinigen sich. Die Schwangere soll Wohlschmeckendes (wie beispielsweise Gerste) und Saures essen. Im zweiten Monat wird mit Shi Gao der Beginn der Fettsubstanz erkennbar. In der Embryonalphase entstehen die Organanlagen aus Ektoderm, Mesoderm und Entoderm. In der Fetalperiode (vom dritten Monat bis zur Geburt) wird das Gesicht des Embryos menschenähnlicher und die Gliedmassen erreichen ihre Länge in Relation zum übrigen Körper. Der dritte und vierte Monat zeigen die Wandlungsphase Feuer (He/Dü), sichtbar in Shi Tai. Der werdenden Mutter wird empfohlen, ungeschälten Reis oder Suppen mit Fisch zu essen sowie den Körper ruhig zu halten. Der vierte und fünfte Monat sind geprägt von starkem Längenwachstum und den Bewegungen des Kindes, die die Mutter wahrnehmen

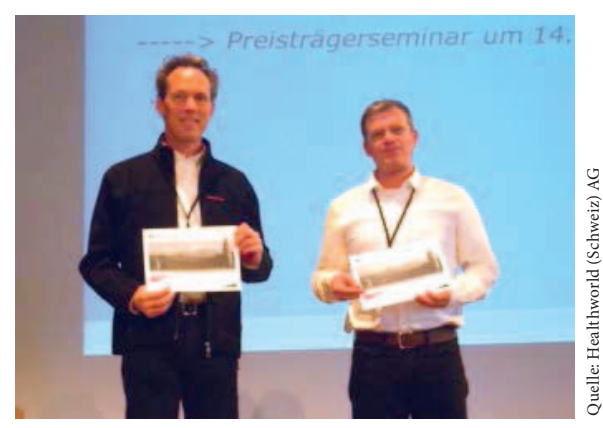

Abb. 2. Grundlagenforschung in der Komplementärmedizin: die Preisträger Stephan Baumgartner, Bern (links), und Tim Jäger, Bern (rechts). 
kann. Mit der Wandlungsphase Erde (MP/Ma) im fünften und sechsten Monat wird der Einfluss auf psychische, soziale, intellektuelle und emotionale Entwicklung sowie die Konstitution manifest. Die Schwangere soll spät aufstehen, warme Unterkleider tragen und Reis, Weizen und Suppen mit Schaf und Rind zu sich nehmen, damit sie das Qi pflegen und so die fünf Zang festigen kann. Vom sechsten bis zum neunten Monat stehen die Gewichtszunahme, die Bildung des Unterfettgewebes sowie die Reifung der Organe (insbesondere der Lungen) im Vordergrund. Der
Schwangeren wird empfohlen, Reisbrei zu essen und sich zu bewegen, damit Qi und Blut zirkulieren, die Knochen genährt und die Zähne gehärtet werden. Mit der Wandlungsphase Wasser $(\mathrm{Ni} / \mathrm{Bl})$ wird im neunten und zehnten Monat die Lebensessenz bereitgestellt. Haut, Körperhaare, die sechs FU-Organe und die hundert Gelenke bilden sich vollständig aus. Im zehnten Monat sind auch die Zang-Organe vollständig ausgebildet. Die in der Schwangerschaft verbotenen Akupunktur-Punkte werden mit diesen Ausführungen gut verständlich.
Im Mittagsintermezzo begegneten sich Ost und West in «Tai Ji trifft Alphorn», und am Nachmittag wurden Stephan Baumgartner und Tim Jäger (Abb. 2) für ihre «Grundlagenforschung in der Komplementärmedizin am Beispiel homöopathischer Arzneimittel» ausgezeichnet.

Im Anschluss an die beiden Kongresstage fand die ASA-Delegiertenversammlung statt. Den Abschluss dieser äusserst spannenden Tagung bildeten die beiden Samstagskurse «Meet the Expert» mit Stephen Birch (Oslo) und Frank He (Santa Cruz, USA). 